\title{
Elementos de la gestión de mantenimiento en las unidades de bombeo mecánico convencionales
}

\author{
Elements of maintenance management in conventional mechanical pumping units
}

Daryeling Betancourt

daryeling@gmail.com

Código ORCID: 0000-0002-1558-9650

Universidad del Zulia. Maracaibo, Venezuela
< Articulo recibido en julio 2019

< Arbitrado en agosto 2019

< Publicado en enero 2020

\section{Resumen}

Se identificaron los elementos de la gestión de mantenimiento presentes en las unidades de bombeo mecánico convencionales. El estudio fue descriptivo, con diseño no experimental, transeccional, y de campo. La población quedó conformada por los gerentes y supervisores de las unidades de bombeo mecánico convencionales de Petróleos de Venezuela S. A. Para la recolección de datos se aplicó la encuesta, a través de un cuestionario contentivo de 25 ítems, utilizando una escala dicotómica. La validación se realizó mediante el juicio de expertos. La confiabilidad se calculó a través del coeficiente Kuder y Richardson, donde se obtuvo 0,93. El análisis de los datos se realizó mediante las frecuencias registradas. Se concluye del análisis realizado alta presencia en la gestión de mantenimiento analizada, señalando como elementos de la gestión la planificación, programación, ejecución, control y evaluación, es decir, el conjunto de acciones pertinentes para su cumplimiento.

Palabras clave: Control, ejecución, elementos de la gestión de mantenimiento, evaluación, planificación, programación

Abstract

The elements of maintenance management present in conventional mechanical pumping units were identified. The study was descriptive, with a non-experimental, transectional, and field design. The population was made up of the managers and supervisors of the conventional mechanical pumping units of Petróleos de Venezuela S. A. For the data collection, the survey was applied through a questionnaire containing 25 items, using a dichotomous scale. Validation was performed through expert judgment. Reliability was calculated using the Kuder and Richardson coefficient, where 0.93 was obtained. Data analysis was performed using the recorded frequencies. It is concluded from the analysis carried out high presence in the maintenance management analyzed, indicating as planning elements, planning, organization, programming, execution, control and evaluation, that is to say, the set of pertinent actions for its fulfillment.

Keywords: Control, execution, elements of maintenance management, evaluation, planning, programming 
INTRODUCCIÓN

La época actual, debido a las consideraciones demandadas por el mercado, se encuentra en un estado de transición en la que la excelencia es considerada parte del producto, por ello el mantenimiento, es función importante de apoyo a la producción, y por ende parte de la organización empresarial. Eventualmente, las empresas tienen latente el reto de cómo mejorar sus actividades de gestión del mantenimiento para ser más sostenibles. Es importante recordar que la sostenibilidad incorpora dos (02) factores: ambiente y subsistencia de la organización, aunado al compromiso social.

Así mismo el mantenimiento como estructura, es un centro de costos a efectos de los intereses de la empresa. Ciertamente, como un costo sólo se justifica si "perfecciona" el negocio, mejorando las condiciones de productividad, mediante la capacidad continúa para adaptarse, desarrollarse y conservarse (independientemente de sus funciones particulares). Para ello, se debe enfocar adecuadamente la visión y misión mediante la definición clara de políticas, objetivos, valores, entre otros.

Además, en los escenarios de hoy, las empresas se juegan su capacidad competitiva por la cantidad y calidad de los recursos que se comprometen en el área de mantenimiento, debido a la capacidad de ésta para generar beneficios a su más inmediato grupo de interés como es, el área de producción. La principal ventaja que ofrece el mantenimiento, reside en la consecución de que los sistemas productivos continúen desempeñando las funciones deseadas y de esta forma contribuir a conservar las actividades productivas, de las cuáles la empresa obtiene las utilidades económicas (produciendo su sostenibilidad en un negocio particular).

Lo anterior da lugar a establecer la gestión del mantenimiento como parámetro de referencia para evaluar, a través de la supervisión de la planificación, ejecución y control, el conjunto de actividades propias de la función, que permiten el uso efectivo y eficaz de los recursos con que cuenta la organización, para alcanzar los objetivos que satisfacen los requerimientos de los diferentes grupos de interés, cuyo fin básico consiste en incrementar la disponibilidad de los sistemas productivos, partiendo de la ejecución de los mismos, mediante las mejoras incrementales a bajo costo, para ser competitivo, logrando que funcionen de forma eficiente y confiable dentro de un contexto de operación.

En este sentido, para Rodríguez (2008) la gestión de mantenimiento implica disponer de metodologías que involucren sistemas de información y una acción cíclica de mejora constituida por auditorías de los puntos críticos de éxito, planificación a la medida y ejecución del plan de trabajo definido aplicando las herramientas de gestión apropiadas.

Por lo tanto, es necesario gestionar correctamente las necesidades $y / 0$ prioridades de la función de mantenimiento, para lograr los resultados adecuados, a través de la mejora en cuanto a la eficacia y eficiencia de procesos para alcanzar la excelencia operativa, cuyo fundamento básico se refiere a ofrecer servicios a un precio competitivo mediante el equilibrio entre la calidad y la funcionalidad siendo la idea principal brindar el mejor costo total.

Lo antes planteado refleja la importancia de la gestión de mantenimiento en las 
empresas, las cuales deben estar sustentadas sobre la base de una planificación, organización, programación, ejecución, control y evaluación, donde el personal constituye una clave determinante para el mejoramiento de los procesos, razón por la cual, se requiere un compromiso de cada uno de los integrantes del sector analizado, en este caso, las unidades de bombeo mecánico convencionales en Petróleos de Venezuela división Costa Oriental del Lago, el cual requiere de una evaluación de su gestión, para crear un entorno ajustado a los lineamientos establecidos.

En este contexto, y según entrevistas no estructuradas realizadas al personal de mantenimiento de las unidades de bombeo mecánico convencionales, se pudo conocer que estos pozos suelen presentar problemas en cuanto a la vida útil del equipo de levantamiento, lo que origina entradas de máquinas imprevistas que interrumpen el proceso de producción y aumentan los gastos operacionales y producción diferida en las diferentes unidades de producción. Asimismo, la falla de este equipo se traduce en múltiples inconvenientes, principalmente en cuanto a importantes pérdidas económicas por la parada de producción de los pozos y gastos imprevistos para la atención de las situaciones presentadas, adicionando la solicitud de personal para prestar dichos servicios.

De acuerdo con lo anterior, el estudio tiene como finalidad identifican los elementos de la gestión de mantenimiento presentes en las unidades de bombeo mecánico convencionales en la Costa Oriental del Lago del sector petrólero.

\section{Elementos de la gestión de mantenimiento}

De acuerdo a Martínez (2007), los elementos de la gestión de mantenimiento, como toda gestión organizacional, están relacionados a un sistema de dirección que se base en la planificación, organización, programación, control y ejecución. Bajo esta óptica la gestión de mantenimiento es un ciclo que se da en forma secuencial, cuyo proceso consta de cuatro elementos básicos: planificar, organizar, controlar y ejecutar. Este ciclo permite realizar una retroalimentación a fin de permitir una mejora continua.

Por otra parte, la gestión del mantenimiento conforma el conjunto de tareas realizadas por el usuario para mantener la funcionabilidad del sistema durante su vida operativa (García, 2009). Visto así $y$, conociendo el significado del término proceso, puede inferirse que el proceso de mantenimiento está compuesto por los elementos que deberán llevarse a cabo para aplicarlo, y éstos deberán estar estrechamente vinculados unos con otros, generando retroalimentación de la información que se genera en cada una de ellos. Cada elemento aporta información para la continuidad de la siguiente.

En este orden, Márquez (2010) define los elementos de la gestión de mantenimiento como el trabajo de planificar, organizar, programar, ejecutar, controlar y evaluar, a realizarse para maximizar tanto la disponibilidad como efectividad de la infraestructura requerida por el sistema de producción. De acuerdo a este autor, el propósito de los elementos es mejorar la funcionalidad de los componentes de equipos o maquinarias en función de la filosofía de las empresas en cuanto a costos, calidad, momento oportuno, lugar apropiado; con la adopción de medidas así como la realización de acciones que permitan alcanzar los objetivos y efectividad de los procesos. 
En este marco de referencia, para Newbrough (2005) en una gestión de mantenimiento, la planificación y programación representan el punto de partida. Indica este autor que, estos dos (2) elementos de la gestión llevan involucrados la necesidad de imaginar y relacionar las actividades probables que habrán de cumplirse para lograr los objetivos de mantenimiento y resultados esperados. Este autor, de manera amplia, describe los elementos de la gestión de mantenimiento como planificación, programación, ejecución, evaluación y control.

Adicionalmente, Prando (2006) concibe la gestión de mantenimiento como un proceso integral basado en normas $y$ procedimientos, en el cual se distribuyen las acciones de la gerencia de línea. Se enfatiza que el mantenimiento como proceso se realiza a nivel operativo, pero como gestión se sustenta en la alta y media gerencia a través de los procesos de planificación, control y supervisión.

Bajo esta definición, la gestión conlleva un proceso administrativo, entendido este, como la base de una estructura en la cual los conocimientos que poseen los gerentes pueden ser organizados en forma útil $y$ práctica. En este sentido, este autor establece los siguientes elementos en la gestión, como son: planificación, organización, integración, ejecución, evaluación y control.

En el marco de estos elementos, la investigadora considera que el ejercicio de estas funciones por parte de los gerentes es de forma más o menos simultánea y no siguiendo un rígido orden preestablecido, puesto que, en la gestión de mantenimiento, el papel de los gerentes como líderes del proceso exige el desempeño de habilidades técnicas, interpersonales, de conceptualización y comunicación. Así, los gerentes deben adaptarse a cambios generados por la diversidad, calidad y pactos comerciales nacionales e internacionales con el fin de lograr los objetivos organizacionales a través de una adecuada gestión de mantenimiento.

Con base en las definiciones antes señaladas, para efectos de esta investigación, se definen los elementos de la gestión de mantenimiento como el conjunto de acciones pertinentes para su cumplimiento, que contribuyan a la efectividad de los procesos productivos, habiendo definido estas acciones como: planificación, programación, ejecución, control y evaluación. A continuación se pasa a explicar cada uno de estos elementos, que conforman los indicadores de esta dimensión.

\section{Planificación}

Para Newbrough (2005) la planificación en la gestión de mantenimiento es un proceso que consiste en la definición de rutinas y procedimientos y en la elaboración de planes detallados para horizontes relativamente largos, usualmente trimestrales o anuales. Implica la determinación de las operaciones necesarias, mano de obra requerida, materiales a emplear, equipos a utilizar y duración de las actividades de mantenimiento.

En tal sentido, en la planificación del mantenimiento, según lo expresa el autor citado, se debe considerar los siguientes aspectos:

- Establecimiento de objetivos y metas en cuanto a los objetos a mantener.

- Garantizar la disponibilidad de los equipos o sistemas. 
- Establecer un orden de prioridades para la ejecución de las acciones.

- Sistema de señalización y codificación lógica.

- Inventario técnico.

- Procedimientos y rutinas de mantenimiento.

- Registros de fallas y causas.

- Estadísticas de tiempo de parada y tiempo de reparación.

En este marco de referencia, Perozo (2007) afirma que la planificación de la gestión de mantenimiento consiste en definir en términos cuantitativos, lógicos, todos los elementos de la gestión, su método de aplicación y secuencia de actividades. Duffuaa y otros (2010) la exponen como el proceso de asignación de recursos y personal para los trabajos a realizar en determinado momento.

Por su parte, Souris (2005) define la planificación en la gestión del mantenimiento como las actividades que se realizan en el marco del sistema de operaciones de producción asociadas al análisis de información, definición de necesidades, objetivo o metas, planificación y programación de actividades, definición de recursos: personal, material, espacio y tiempo. La función de planificación debe establecer tanto objetivos como metas, en cuanto a necesidades y tiempo para ejecutar las acciones requeridas, que garanticen disponibilidad de los sistemas, todo incluido en forma detallada en un plan.

En un sentido más amplio, Márquez (2010) define la planificación en la gestión del mantenimiento como un proceso integral y continuo que se realiza para determinar los objetivos, recursos y métodos que se necesitan en los procesos de mantenimiento para lograr sus fines. El autor citado afirma que para la planificación del mantenimiento se debe tener en cuenta lo que desean los clientes internos y externos, los recursos que se van a emplear, la tecnología y procedimientos a manejar en las diferentes tareas, cómo se va a medir y controlar el desempeño de mantenimiento y sus procesos; $y$, finalmente como se podría mejorar la eficiencia, productividad $y$ calidad.

Por otro lado, la Norma COVENIN 2500-93 establece que, se debe definir una política general por parte de la gerencia de mantenimiento que involucre su campo de acción, justificación, medios y objetivos que se persiguen. Se debe tener una planificación para la ejecución de cada una de las acciones de mantenimiento, utilizando los recursos disponibles. La organización debe contar con un sistema de señalización o codificación lógica y secuencial que permita registrar información del proceso o de cada línea, máquina o equipo en el sistema total. Se debe elaborar un inventario técnico de cada sistema: ubicación, descripción y datos; para elaborar los planes de mantenimiento.

En tal sentido, Adam y Ebert (2006) exponen que los sistemas de mantenimiento se ponen en movimiento por las órdenes de trabajo, que generalmente son emitidas por los departamentos de producción. Estas órdenes de trabajo describen el trabajo, su ubicación, habilidades requeridas y prioridades del trabajo. Según su finalidad o uso, la orden de trabajo puede asumir diferentes nombres y formatos.

En algunos casos, la orden de trabajo adquiere las funciones de solicitud de intervención, para que se realice una 
actividad de mantenimiento correctivo, 0 preventivo programado, o tal vez de inspección o mantenimiento rutinario, o para realizar algunas actividades preparatorias. También puede asumir el papel de orden de fabricación o recuperación de piezas para el taller, o servir de vehículo a disposiciones de carácter normativo. Dichos autores establecen que la planificación de un sistema debe incluir el diseño del trabajo, estándares de tiempo y administración de proyectos.

- Diseño del trabajo: en lo que se refiere a mantenimiento comprende el contenido de trabajo de cada tarea y determina el método que se va a utilizar, las herramientas especiales necesarias y los trabajadores calificados requeridos.

- Estándares de tiempo: una vez que la tarea de mantenimiento ha pasado por la etapa de diseño, es básico estimar el tiempo necesario para completar el trabajo. Los estándares de tiempo realistas representan un elemento muy valioso para vigilar e incrementar la eficacia de los trabajadores y de esta forma reducir al mínimo el tiempo muerto en los procesos. No es esencial tener estándares de tiempos para todos los trabajos de mantenimiento, solo para aquellos que son críticos, consumen mucho tiempo o representan un cuello de botella.

- Administración de proyectos: en el caso de plantas o procesos grandes las reparaciones generales de gran envergadura $\circ$ el mantenimiento preventivo que se han planificado, se hacen periódicamente. Durante estos trabajos toda la planta se paraliza, en tal sentido es necesario tener en mente minimizar el tiempo muerto, por lo que es necesario planificar y graficar el trabajo de tal manera que se garantice el mejor uso de los recursos.

Por otro lado, para Neto (2008) la planificación permite programar los proyectos a mediano y largo plazo de las acciones de mantenimiento que dan la dirección a la industria. Los beneficios alcanzados al llevar un plan, son menor consumo de horas hombre, disminución de inventarios, menor tiempo de parada de equipos, mejora el clima laboral en el personal de mantenimiento, mejora la productividad (eficiencia $x$ eficacia) y ahorro en costos.

Desde la óptica de la investigadora, la planificación es importante ya que contribuye con el logro de los objetivos y propósitos del mantenimiento, ayuda a contrarrestar la incertidumbre y el cambio, facilitando el control. Adicionalmente, esta función primaria sobre la cual se desarrollan y ejecutan las otras funciones, tiene un carácter constante cuando a partir de los planes iníciales que se deben llevar a cabo para emprender el trabajo de la organización, se tiene necesidad de hacer planes adicionales para atender nuevas situaciones que van surgiendo $y$, además, debe revisarse continuamente para hacer los ajustes que se impongan por la dinámica misma de la organización.

\section{Programación}

Una vez terminada la planificación del mantenimiento, donde se consideran la determinación de los recursos necesarios para llevar adelante las funciones del mantenimiento, se procede a la programación del mantenimiento, la cual 
consiste en el proceso de asignación de recursos y personal para los trabajos que tienen que realizarse en ciertos momentos (Duffuaa y otros, 2010).

En el manual de mantenimiento de PDVSA (2005), se define la programación en la gestión de mantenimiento como las acciones necesarias para garantizar la ejecución de las actividades del plan en función de tiempo y recursos. Es donde se sincronizan las actividades diarias, semanales, adicionalmente se coordina el suministro de materiales e insumos necesarios para las actividades, el registro de información de costos y estadísticas de todas las actividades ejecutadas, atención de emergencias, coordinación de guardias, disponibilidad del personal, recursos, así como empresas subcontratistas que sean necesarias para cubrir las eventualidades.

De acuerdo a Newbrough (2005), el proceso de programación consiste en establecer frecuencias para las asignaciones del mantenimiento, las fechas programadas son esenciales para que exista una continua disponibilidad de equipos e instalaciones, se inicia con la solicitud y envió de la orden de trabajo. Por su parte Adam y Ebert (2006), consideran que la programación del mantenimiento permitirá saber con exactitud que herramientas, repuestos, materiales se debe disponer antes de emprender una tarea, en conjunto con las herramientas de gestión técnica. Dentro de las actividades de organización, pueden diseñarse fichas para cada caso de mantenimiento predictivo, preventivo o correctivo.

Desde esta perspectiva, Duffuaa y otros (2010) afirman que es necesario asegurar que los trabajadores, piezas y materiales requeridos estén disponibles antes de poder programar una tarea. En la programación de mantenimiento deben tomarse en cuenta los equipos críticos de una planta o proceso productivo, ya que una falla de este equipo detendrá el proceso de producción o pondrá en riesgo la seguridad de los trabajadores.

Estos autores, expresan que el trabajo de mantenimiento para estos equipos se maneja bajo prioridades y es atendido previamente al iniciar cualquier otro trabajo. Motivado a que la ocurrencia de tales trabajos no puede predecirse con certeza, en estos casos, los programas para el mantenimiento planeado deben ser revisados. La eficiencia del programa va a depender primeramente si es flexible, es decir, se ajusta a los cambios.

Así mismo, la investigadora considera que es importante fijar estándares de tiempo para la ejecución de cada uno de los trabajos con el objetivo de aumentar la eficiencia en los trabajadores y reducir el tiempo muerto. Estas acciones de programación incluirán actividades de supervisión, evaluación y dirección del personal, el registro de trabajos realizados, planificación de trabajos, para mejor resultado se debe utilizar un software permitiendo mejorar y optimizar la programación en general.

\section{Ejecución}

Para Dounce (2008), ejecutar significa "poner por obra una cosa", por lo que desde el punto de vista administrativo, la ejecución es una acción del administrador para que sus subordinados se propongan a alcanzar los objetivos establecidos en la planificación y estructurados por la organización. Para llevar a cabo este punto es necesario valerse de los siguientes puntos motivación, comunicación, dirección y coordinación.

Ahora bien, la ejecución en la gestión de mantenimiento es definida por Newbrough (2005), como el proceso mediante el cual se 
ponen en marcha las acciones previstas en la planificación, por consiguiente implica asegurar el cumplimiento de los objetivos previstos a través de la aplicación en el sistema de las actividades métodos y técnicas con los recursos disponibles. La cantidad de mantenimiento está relacionada con el uso de los equipos en el tiempo, por la carga y manejo de los mismos.

Por su parte, Souris (2005) establece las diferencias entre planificar y programar con respecto a la ejecución, indicando que la ejecución es la fase operativa del proceso de gestión del mantenimiento, mientras que los primeros son más de tipo intelectual. Las actividades de ejecución, involucran a todos los gerentes y empleados, mientras que las fases anteriores posiblemente sólo impliquen a pocos gerentes de alto nivel.

De manera similar, Márquez (2010) define la ejecución como la parte del proceso de la gestión de mantenimiento en la cual se realizan los trabajos de mantenimiento tanto programados como de emergencia, consiste en la realización del trabajo diario, que implica: suministro de materiales y equipos, seguridad del trabajo diario, medición, registro de datos, supervisión y seguimiento del trabajo diario.

Al respecto, afirma Moreno (2012), para la ejecución de actividades de mantenimiento, se debe implementar toda una gama de documentación administrativa y técnica, manual de sistema de gestión, procedimientos administrativos, procedimientos de trabajo, instructivos técnicos, registros administrativos, registros de mantenimiento, registro de planificación diaria, registros de análisis de fallas, entre otros.

Acota Moreno (2012) que, en este proceso se debe activar el control del mantenimiento, con base en el control de condición de los equipos que se realiza mediante el uso de los sentidos, complementado con el empleo de procedimientos técnicos. En su mayoría, estos procedimientos comprenden una actividad directa de medición o indirecta, lo que puede significar un ensayo de funcionamiento o la observación de una disfunción.

El mantenimiento se realiza según el nivel mínimo permitido de las propiedades cualitativas de cada elemento; nivel máximo de las propiedades cualitativas que deben elevarse; tiempo de uso o funcionamiento durante el cual las propiedades cualitativas bajan del nivel alto al bajo; modo en que los elementos están sometidos a tensión, carga, desgaste, corrosión, entre otros, que causan pérdida de las propiedades cualitativas o de la capacidad de los elementos para resistirlas. En este sentido, las empresas como entidades que buscan el mejoramiento continuo de los diferentes procesos, requieren un manual de mantenimiento para los equipos que operan en él, ya que optimiza su funcionamiento, protege $y$ alarga la vida útil de dichos equipos.

Ante los planteamientos de los autores citados, la investigadora deduce que la ejecución en la gestión de mantenimiento implica realizar acciones de dirección y coordinación de los grupos realizadores de las actividades generadas en los procesos de planificación y programación, cuya finalidad es garantizar el logro de los objetivos propuestos. Es importante acotar que para ejecutar el programa de mantenimiento se requiere elaborar unos reportes de las actividades que se van a ejecutar, por lo general se utilizan las ordenes de trabajo, esto para facilitar el control. En general, estas órdenes permiten que las actividades 
se realicen tal cual fueron planificadas, comparar los resultados con estándares y evaluarlos de forma de retroalimentar el proceso inicial.

\section{Control}

El control es la función administrativa por medio de la cual se evalúa el rendimiento. Se puede expresar que el control es la etapa del proceso que permite la supervisión y comparación de resultados obtenidos contra resultados esperados, asegurando que la acción dirigida se esté llevando a cabo de acuerdo con los planes de la organización y dentro de los límites de la estructura organizacional. El control es una función clave en el mantenimiento, ya que le permite alcanzar su propósito: mantener equipos e instalaciones funcionando, al menor costo y maximizando la productividad.

Por su parte, Newbrough (2005) define la ejecución, control y evaluación indicando que estos procesos vinculan dos (02) acciones administrativas de singular importancia como son la dirección y coordinación de los esfuerzos del grupo de realizadores de las actividades generadas en los procesos de planificación y programación, cuya finalidad es garantizar el logro de los objetivos propuestos.

En específico, las actividades de control como parte esencial de la administración de cualquier sistema en el caso del mantenimiento implica el control de los trabajos, de los inventarios, de los costos y de la calidad, según Adam y Ebert (2006), a fin de garantizar el cumplimiento de los objetivos planeados. Bajo este bosquejo se describe cada aspecto de acuerdo a los postulados de estos autores:
- Control de trabajos: la administración y control de trabajo de mantenimiento son esenciales para lograr los planes establecidos. Tal es el caso del sistema de órdenes de trabajo, esta herramienta se utiliza para controlar el trabajo de mantenimiento. Una orden de trabajo bien diseñada con un adecuado sistema de informes es el corazón de un sistema de este tipo.

- Control de inventarios: el control de inventario es la técnica de mantener repuestos que disminuyan el costo de tener las partes y componentes en existencia y el costo que se incurre si los repuestos no están disponibles. También proporciona la información necesaria para asegurar la disponibilidad de los repuestos necesarios para la oportuna ejecución de los trabajos de mantenimiento. Si no están disponibles los repuestos se deben tomar las acciones para lograr su abastecimiento e informar al departamento de programación la fecha en la cual estarán disponibles.

- Control de costos: es una función de la filosofía de mantenimiento, el patrón de operación, el tipo de sistema, y los procedimientos y normas de la organización. Es un componente importante en el ciclo de vida de los equipos. El control de costos optimiza todos los costos de mantenimiento, logrando al mismo tiempo los objetivos de la organización, como son los niveles de producción, venta, disponibilidad, porcentaje de calidad y otras medidas de eficacia y eficiencia. 
- Control de calidad: se ejerce midiendo los atributos del producto o servicio prestado, comparando éstos con las especificaciones preestablecidas. Una alta calidad se asegura verificando los trabajos crítico o mediante la supervisión del mantenimiento. Lograr la calidad y objetivos de confiabilidad son responsabilidad del personal de mantenimiento. El esfuerzo combinado y dedicación de los involucrados son clave para garantizar la calidad y confiabilidad de las reparaciones.

Otra teoría sobre el control del mantenimiento es la expuesta por Dounce (2008), para quien es la comprobación de que tanto las personas como los recursos físicos y técnicos se estén empleando, según lo planeado en el tiempo considerado, con o sin desviaciones a la norma determinada, este se inicia desde la planificación, continúa durante todo el proceso administrativo, por lo que es dinámico. Para facilitar el control es necesario atender factores como medición, comparación, análisis y corrección.

De lo planteado, la investigadora considera que, el control consiste en medir resultados y verificar con respecto a especificaciones, implica: un proceso de supervisión de las actividades realizadas; utilizar estándares pre-establecidos para determinar posibles desviaciones de resultados; permitir la corrección de errores, de posibles desviaciones en los resultados o en las actividades realizadas; y en último lugar planificar las actividades y objetivos a realizar, después de haber hecho las correcciones necesarias.

Por tanto, si hay un control efectivo se logra conocer el verdadero estado de los bienes, muebles 0 inmuebles, de haber desviaciones, se podrán establecer medidas correctivas para reafirmar cualquier plan, buscando optimizar dicho plan. La importancia que tiene radica en que se logrará precisar si lo realizado se ajusta a lo planeado, y en caso de existir desviaciones, identificar los responsables y corregir dichos errores. Permite estudiar el pasado para determinar lo que ha ocurrido y porque los estándares no han sido alcanzados; de esta manera se pueden adoptar las medidas necesarias para que en el futuro no se cometan los errores del pasado.

\section{Evaluación}

En la evaluación de un sistema de mantenimiento organizado se mide todo el conjunto en general que lo forma. En la gestión de mantenimiento, no solo se controla y evalúan los servicios prestados, sino al personal que constituye la unidad, herramientas, equipos, definiciones e instalaciones, y puede realizarse por medio analítico.

Según la Fundación Educación Industrias Fundei (2009), la gestión de mantenimiento no solo se controla por los movimientos de los servicios prestados, sino también puede medirse por índices que analizados que conducen a la toma de decisiones. Estos índices son formulaciones basadas en el número de órdenes de trabajo, número de órdenes pendientes, avance de los trabajadores en ejecución y trabajos terminados, horas-hombres efectivas y no efectivas, eficiencia porcentual de cada sección.

Por su parte, Acosta (2007) indica que se debe evaluar la eficiencia de la política de mantenimiento que se ha planificado para el entorno productivo de una organización, e 
identificar las áreas en que tiene deficiencias estructurales y circunstanciales, lo cual es un aporte sustancioso puesto que la dirección puede establecer una ruta de acción para erradicar las debilidades detectadas.

Es por ello, que la investigadora opina que la aplicación periódica de instrumentos de evaluación permite detectar cómo responde la organización ante las variaciones a las que está constantemente sujeta y de qué forma debe adecuarse. Es por esto que hoy día las evaluaciones forman parte de revisiones cíclicas y sistemáticas, donde se identifican las desviaciones y se plantean soluciones a las mismas.

\section{MÉTODO}

El estudio fue de tipo descriptivo, con diseño no experimental, transeccional, y de campo. La población quedó conformada por las cinco (5) unidades de bombeo mecánico convencionales ubicadas en los distintos campos de Petróleos de Venezuela S. A. división Costa Oriental del Lago: Tía Juana,
Lagunillas, Bachaquero, Cumarebo y Cabimas. Siendo los sujetos informantes 15 empleados, entre gerentes y supervisores involucrados en estos procesos.

Para la recolección de datos se aplicó la técnica de la encuesta, a través del uso de un cuestionario contentivo de 25 ítems, utilizando una escala dicotómica. La validación del instrumento se realizó mediante el juicio de cinco (5) expertos en el área. La confiabilidad se calculó a través del coeficiente Kuder y Richardson, donde se obtuvo como valor de confiabilidad total del instrumento 0,93, al incluir todos los ítems.

El análisis de los datos se realizó mediante la estadística descriptiva, a través del estudio de las frecuencias registradas. En este sentido, una vez obtenidos los resultados se organizaron agrupándolos por indicadores, para así poder efectuar un análisis de la información de frecuencias a través del baremo de interpretación mostrado en la Tabla 1.

Tabla 1. Baremo de interpretación de la frecuencia

\begin{tabular}{cc}
\hline \multicolumn{2}{c}{ RANGO PARA FRECUENCIA RELATIVA } \\
\hline $\mathrm{SI} \geq 70 \%$ & Alta presencia \\
$40 \% \leq \mathrm{SI}<70 \%$ & Mediana presencia \\
$\mathrm{SI}<40 \%$ & Baja presencia \\
\hline
\end{tabular}

Fuente: Elaboración propia

\section{RESULTADOS}

En la Tabla 2 se muestran los resultados para el indicador planificación, de la dimensión elementos de la gestión de mantenimiento, correspondientes a los ítems $1,2,3,4$ y 5 del instrumento. De acuerdo al baremo establecido, en las unidades de bombeo mecánico convencionales bajo estudio, se asigna alta presencia a la planificación como elemento de la gestión de mantenimiento que desarrollan, situación que se devela al ubicarse en promedio el $89,33 \%$ de respuestas en la opción sí. 
Tabla 2. Indicador: Planificación

\begin{tabular}{|c|c|c|c|c|c|c|c|c|}
\hline \multirow{2}{*}{\multicolumn{2}{|c|}{$\begin{array}{l}\text { Dimensión: } \\
\text { Elementos de la gestión de mantenimiento }\end{array}$}} & \multicolumn{6}{|c|}{$\begin{array}{c}\text { RESPUESTAS / } \\
\text { ESCALA DICOTÓMICA }\end{array}$} & \multirow[b]{3}{*}{ BAREMO } \\
\hline & & \multicolumn{2}{|r|}{$\mathrm{SI}$} & \multicolumn{2}{|c|}{ NO } & \multicolumn{2}{|c|}{ Total } & \\
\hline $\mathrm{N}^{\circ}$ & Ítem & $\mathrm{Fa}$ & $\mathrm{Fr}$ & $\mathrm{Fa}$ & $\mathrm{Fr}$ & $\mathrm{n}$ & $\%$ & \\
\hline 1 & $\begin{array}{l}\text { ¿La planificación del mantenimiento se } \\
\text { realiza con holgura del tiempo de } \\
\text { ejecución? }\end{array}$ & 10 & 66,67 & 5 & 33,33 & 15 & 100 & Moderada presencia \\
\hline 2 & $\begin{array}{l}\text { ¿Determinan el personal requerido para } \\
\text { la ejecución del mantenimiento en la } \\
\text { planificación? }\end{array}$ & 13 & 86,67 & 2 & 13,33 & 15 & 100 & Alta presencia \\
\hline 3 & $\begin{array}{l}\text { ¿̇Antes de iniciar el mantenimiento se } \\
\text { determinan los materiales que se } \\
\text { requieren para llevarlo a cabo? }\end{array}$ & 14 & 93,33 & 1 & 6,67 & 15 & 100 & Alta presencia \\
\hline 4 & $\begin{array}{l}\text { ¿Previa a la ejecución del mantenimiento } \\
\text { se determinan las herramientas } \\
\text { requeridas para realizarlo? } \\
\text { ¿En el plan del mantenimiento se }\end{array}$ & 15 & 100 & 0 & 0,00 & 15 & 100 & Alta presencia \\
\hline \multirow[t]{2}{*}{5} & $\begin{array}{l}\text { definen las metas que se desean } \\
\text { alcanzar? }\end{array}$ & 15 & 100 & 0 & 0,00 & 15 & 100 & Alta presencia \\
\hline & Totales & 13 & 89,33 & 2 & 10,67 & 15 & 100 & Alta presencia \\
\hline
\end{tabular}

Fuente: Elaboración propia

Estos resultados reflejan que, en las unidades de bombeo analizadas, las siguientes actividades ostentan alta presencia: en la planificación determinan el personal requerido para la ejecución del mantenimiento $(86,67 \%)$; antes de iniciar el mantenimiento determinan los materiales que se requieren para llevarlo a cabo (93,33\%); previa a la ejecución del mantenimiento determinan las herramientas requeridas para realizarlo (100\%); y en el plan del mantenimiento definen las metas que se desean alcanzar (100\%). No obstante, se categoriza con moderada presencia el que la planificación del mantenimiento se realice con holgura del tiempo de ejecución (66,67\%).

Los resultados evidenciados validan los postulados teóricos de Souris (2005), para quien la planificación en la gestión del mantenimiento es el conjunto de actividades que se realizan en el marco del sistema de operaciones de producción asociadas al análisis de la información, definición de las necesidades, objetivo o metas, planificación y programación de actividades, definición de recursos: personal, material, espacio y tiempo. La función de planificación debe establecer tanto objetivos como metas, en cuanto a necesidades y tiempo para ejecutar las acciones requeridas, que garanticen disponibilidad de los sistemas, todo incluido en forma detallada en un plan.

A juicio de la investigadora, las unidades de bombeo mecánico convencionales bajo estudio tienen presente la importancia de la planificación en la gestión de mantenimiento que llevan a cabo, reconociendo que ésta contribuye con el logro de los objetivos y propósitos del mantenimiento, ayudando a contrarrestar la incertidumbre y el cambio, facilitando el control. 
Los resultados mostrados en la tabla 3 corresponden al indicador programación, de la dimensión elementos de la gestión de mantenimiento. Se aprecia que, en promedio, el $65,33 \%$ de los encuestados consideran que las actividades involucradas a los ítems se realizan con moderada presencia. Esta situación es explicada por los niveles de presencia alcanzados en los ítems involucrados al indicador.

Tabla 3. Indicador: Programación

\begin{tabular}{|c|c|c|c|c|c|c|c|c|}
\hline \multirow{3}{*}{\multicolumn{2}{|c|}{$\begin{array}{l}\text { Dimensión: } \\
\text { Elementos de la gestión de mantenimiento } \\
\text { № Ítem }\end{array}$}} & \multicolumn{6}{|c|}{$\begin{array}{c}\text { RESPUESTAS / } \\
\text { ESCALA DICOTÓMICA }\end{array}$} & \multirow[b]{3}{*}{ BAREMO } \\
\hline & & \multicolumn{2}{|r|}{$\mathrm{SI}$} & \multicolumn{2}{|c|}{ NO } & \multicolumn{2}{|c|}{ Total } & \\
\hline & & $\mathrm{Fa}$ & $\mathrm{Fr}$ & $\mathrm{Fa}$ & $\mathrm{Fr}$ & $\mathrm{n}$ & $\%$ & \\
\hline 6 & $\begin{array}{l}\text { ¿Aplican programas de trabajo para } \\
\text { ejecutar el mantenimiento? } \\
\dot{2} \text { Utilizan un software computarizado para }\end{array}$ & 13 & 86,67 & 2 & 13,33 & 15 & 100 & Alta presencia \\
\hline 7 & $\begin{array}{l}\text { efectuar la programación de } \\
\text { mantenimiento? }\end{array}$ & 5 & 33,33 & 10 & 66,67 & 15 & 100 & Baja presencia \\
\hline 8 & $\begin{array}{l}\text { ¿̇lncluyen la asignación de recursos en el } \\
\text { programa de trabajo? }\end{array}$ & 11 & 73,33 & 4 & 26,67 & 15 & 100 & Alta presencia \\
\hline 9 & $\begin{array}{l}\text { ¿Contemplan las prioridades de los equipos } \\
\text { o situación requerida de mantenimiento en } \\
\text { el programa del mismo? }\end{array}$ & 12 & 80 & 3 & 20,00 & 15 & 100 & Alta presencia \\
\hline \multirow[t]{2}{*}{$\begin{array}{l}1 \\
0\end{array}$} & $\begin{array}{l}\text { ¿Asignan los recursos disponibles para que } \\
\text { los trabajos se realicen en el tiempo } \\
\text { planeado? }\end{array}$ & 8 & 53 & 7 & 46,67 & 15 & 100 & $\begin{array}{l}\text { Moderada } \\
\text { presencia }\end{array}$ \\
\hline & Totales & 10 & 65,33 & 5 & 34,67 & 15 & 100 & $\begin{array}{l}\text { Moderada } \\
\text { presencia }\end{array}$ \\
\hline
\end{tabular}

Fuente: Elaboración propia

Así las cosas, un $86,67 \%$ respondieron de manera afirmativa que se aplican programas de trabajo para ejecutar el mantenimiento; para el $73,33 \%$ se incluye la asignación de recursos en el programa de trabajo; y para el $80 \%$ se contemplan las prioridades de los equipos o situación requerida de mantenimiento en el programa del mismo; de tal forma que estas actividades fueron categorizadas con alta presencia. No obstante, se muestra moderada presencia en cuanto a si asignan los recursos disponibles para que los trabajos se realicen en el tiempo planeado, con 53\% de respuestas afirmativas; y baja presencia en lo concerniente a si utilizan un software computarizado para efectuar la programación de mantenimiento, en el $33,33 \%$ de los casos.
Dado los resultados mostrados, se evidencia mediana congruencia con Newbrough (2005), para quien el proceso de programación consiste en establecer frecuencias para las asignaciones del mantenimiento, las fechas programadas son esenciales para que exista una continua disponibilidad de equipos e instalaciones. También coinciden medianamente con Adam y Ebert (2006), cuando consideran que la programación del mantenimiento permitirá saber con exactitud de que recursos, repuestos, materiales o herramientas se debe disponer antes de emprender una tarea, en conjunto con las herramientas de gestión técnica.

Con base a lo expuesto, a criterio de la investigadora, la programación no está 
siendo considerada como punta de lance en el proceso de mantenimiento llevado a cabo en las unidades de bombeo mecánico convencionales analizadas, dado su nivel de moderada presencia, evidenciándose como una oportunidad de mejora en esta gestión. Es necesario que reconozcan la importancia de fijar estándares de tiempo para la ejecución de cada uno de los trabajos, con el objetivo de aumentar la eficiencia en los trabajadores y reducir el tiempo muerto. De igual manera, se deben considerar acciones de programación que incluyan actividades de supervisión, evaluación y dirección del personal, registro de trabajos realizados, planificación de trabajos, para mejor resultado utilizar un software permitiendo mejorar y optimizar la programación en general.

En la Tabla 4 se muestran los valores obtenidos de las respuestas correspondientes a los ítems 11,12, 13, 14 y 15 relacionados al indicador ejecución. Se observa que el $80 \%$ de los sujetos encuestados respondieron de manera afirmativa a las proposiciones emitidas, indicando que las actividades relacionadas a los ítems se realizan con alta presencia, de acuerdo al baremo utilizado para tal fin.

Tabla 4. Indicador: Ejecución

\begin{tabular}{|c|c|c|c|c|c|c|c|c|}
\hline \multirow{2}{*}{\multicolumn{2}{|c|}{$\begin{array}{l}\text { Dimensión: } \\
\text { Elementos de la gestión de mantenimiento }\end{array}$}} & \multicolumn{6}{|c|}{$\begin{array}{c}\text { RESPUESTAS / } \\
\text { ESCALA DICOTÓMICA }\end{array}$} & \multirow[b]{3}{*}{ BAREMO } \\
\hline & & \multicolumn{2}{|r|}{ SI } & \multicolumn{2}{|c|}{ NO } & \multicolumn{2}{|c|}{ Total } & \\
\hline $\mathrm{N}^{\circ}$ & Ítem & $\mathrm{Fa}$ & $\mathrm{Fr}$ & $\mathrm{Fa}$ & $\mathrm{Fr}$ & $\mathrm{n}$ & $\%$ & \\
\hline 11 & $\begin{array}{l}\text { ¿Es suficiente el recurso humano } \\
\text { asignado para ejecutar cada } \\
\text { mantenimiento en el tiempo requerido? }\end{array}$ & 8 & 53,33 & 7 & 46,67 & 15 & 100 & Moderada presencia \\
\hline 12 & $\begin{array}{l}\text { ¿Asignan el recurso humano calificado } \\
\text { para ejecutar cada mantenimiento? }\end{array}$ & 14 & 93,33 & 1 & 6,67 & 15 & 100 & Alta presencia \\
\hline 13 & $\begin{array}{l}\text { ¿Ejecutan según un orden de prioridad } \\
\text { establecido las actividades de } \\
\text { mantenimiento? }\end{array}$ & 14 & 93,33 & 1 & 6,67 & 15 & 100 & Alta presencia \\
\hline 14 & $\begin{array}{l}\text { ¿Cumplen con las metas planificadas en } \\
\text { la ejecución de los mantenimientos? }\end{array}$ & 10 & 66,67 & 5 & 33,33 & 15 & 100 & Moderada presencia \\
\hline \multirow[t]{2}{*}{15} & $\begin{array}{l}\text { ¿Se siguen normas pre-establecidas al } \\
\text { ejecutar las actividades de } \\
\text { mantenimiento? }\end{array}$ & 14 & 93,33 & 1 & 6,67 & 15 & 100 & Alta presencia \\
\hline & Totales & 12 & 80,00 & 3 & 20,00 & 15 & 100 & Alta presencia \\
\hline
\end{tabular}

Fuente: Elaboración propia

Al detallar estos resultados se aprecian tres actividades catalogadas con alta presencia, en específico las medidas por los ítems 12, 13 y 15, en los cuales para el $93,33 \%$ de los encuestados se asigna el recurso humano calificado para ejecutar cada mantenimiento, ejecutan según un orden de prioridad establecido las actividades de mantenimiento, y siguen normas preestablecidas al ejecutar las actividades de mantenimiento. No obstante, al consultarles si es suficiente el recurso humano asignado 
para ejecutar cada mantenimiento en el tiempo requerido, el 53,33\% respondió de manera afirmativa demarcando esta situación con moderada presencia.

Los resultados, de alta presencia, validan lo expuesto por Márquez (2010), para quien la ejecución es la parte de la gestión de mantenimiento en la cual se realizan los trabajos tanto programados como de emergencia, consiste en la realización del trabajo diario, que implica: suministro de materiales y equipos, seguridad del trabajo diario, medición, registro de datos, supervisión y seguimiento del trabajo diario.

A juicio de la investigadora, en las unidades de bombeo mecánico convencionales bajo análisis, se está realizando de forma satisfactoria la ejecución del mantenimiento en lo referente a realizar las acciones de dirección y coordinación de los grupos realizadores de las actividades generadas en los procesos de planificación y programación, garantizando el logro de los objetivos propuestos.

La Tabla 5 muestra las respuestas del indicador control, de la dimensión bajo análisis, en la cual se aprecia que para el $80 \%$ de los encuestados, en promedio, en las unidades de bombeo bajo estudio se tiene alta presencia de las actividades relacionadas al indicador. Esta situación es explicada por el comportamiento de las respuestas afirmativas dadas a cada ítem involucrado.

Tabla 5. Indicador: Control

\begin{tabular}{|c|c|c|c|c|c|c|c|c|}
\hline & $\begin{array}{l}\text { Dimensión: } \\
\end{array}$ & & $\begin{array}{r}R \\
\text { ESCAL }\end{array}$ & $\begin{array}{l}\text { ESPUI } \\
\text { A DIC }\end{array}$ & $\begin{array}{l}\text { ESTAS / } \\
\text { COTÓN }\end{array}$ & ICA & & \\
\hline & ementos de ia gesiton ae manienimienio & & SI & & NO & & tal & \\
\hline $\mathrm{N}^{\circ}$ & Ítem & $\mathrm{Fa}$ & $\mathrm{Fr}$ & $\mathrm{Fa}$ & $\mathrm{Fr}$ & $\mathrm{n}$ & $\%$ & BAREMO \\
\hline & $\dot{z}$ Utilizan órdenes de trabajo como & & & & & & & \\
\hline 16 & sistema de control para el seguimiento & 13 & 86,67 & 2 & 13,33 & 15 & 100 & Alta presencia \\
\hline & de los trabajos de mantenimiento? & & & & & & & \\
\hline & ¿̇Se compara el porcentaje de & & & & & & & \\
\hline 17 & $\begin{array}{l}\text { mantenimiento realizado con el } \\
\text { programado? }\end{array}$ & 14 & 93,33 & 1 & 6,67 & 15 & 100 & Alta presencia \\
\hline & ¿̇Analizan la información registrada & & & & & & & \\
\hline 18 & sobre los mantenimientos realizados & 9 & 60,00 & 6 & 40,00 & 15 & 100 & Moderada presencia \\
\hline & para detectar acciones correctivas? & & & & & & & \\
\hline & ¿̇evisan periódicamente que los costos & & & & & & & \\
\hline 19 & $\begin{array}{l}\text { de mantenimiento estén dentro de lo } \\
\text { previsto? }\end{array}$ & 12 & 80,00 & 3 & 20,00 & 15 & 100 & Alta presencia \\
\hline & ¿ेVerifican que se dispone de inventarios & & & & & & & \\
\hline 20 & $\begin{array}{l}\text { (materiales, repuestos y piezas) para } \\
\text { ejecutar el mantenimiento? }\end{array}$ & 12 & 80,00 & 3 & 20,00 & 15 & 100 & Alta presencia \\
\hline & Totales & 12 & 80,00 & 3 & 20,00 & 15 & 100 & Alta presencia \\
\hline
\end{tabular}

Fuente: Elaboración propia

Así, se categorizaron con alta presencia las actividades que implican: la utilización de órdenes de trabajo como sistema de control para el seguimiento de los trabajos de mantenimiento (86,67\%); el comparar el porcentaje de mantenimiento realizado con el programado (93,33\%); revisar periódicamente que los costos de 
mantenimiento estén dentro de lo previsto $(80 \%) ;$ y verificar que se dispone de inventarios (materiales, repuestos y piezas) para ejecutar el mantenimiento (80\%). Sin embargo, denotaron con moderada presencia el que analicen la información registrada sobre los mantenimientos realizados para detectar acciones correctivas, con el $60 \%$ de respuestas afirmativas.

Los resultados concuerdan con lo establecido por Dunce (2008), para quien el control es la comprobación de que tanto las personas como los recursos físicos y técnicos se estén empleando, según lo planeado en el tiempo considerado, con o sin desviaciones a la norma determinada, este se inicia desde la planificación, continúa durante todo el proceso administrativo, por lo que es dinámico.

En este sentido, a juicio de la investigadora, se confirma que en efecto el indicador control es considerado dentro de la gestión del mantenimiento en las unidades de bombeo bajo análisis, prestando atención a que el mismo consiste en medir resultados y verificar con respecto a las especificaciones, utilizando estándares o patrones establecidos para determinar posibles desviaciones de los resultados; no obstante, la corrección de errores muestra oportunidades de mejora, de manera que se puedan planificar las actividades y objetivos a realizar, después de haber hecho las correcciones necesarias.

En la Tabla 6 se muestran los resultados para el indicador evaluación, último indicador considerado en la dimensión elementos de la gestión de mantenimiento. De acuerdo al baremo, en las unidades de bombeo bajo estudio, se asigna moderada presencia a la evaluación como elemento de la gestión de mantenimiento que desarrollan, situación que se devela al ubicarse, en promedio, el 52\% de respuestas en la opción sí.

Tabla 6. Indicador: Evaluación

\begin{tabular}{|c|c|c|c|c|c|c|c|c|}
\hline \multirow{3}{*}{\multicolumn{2}{|c|}{$\begin{array}{l}\text { Dimensión: } \\
\text { Elementos de la gestión de mantenimiento }\end{array}$}} & \multicolumn{6}{|c|}{$\begin{array}{c}\text { RESPUESTAS / } \\
\text { ESCALA DICOTÓMICA }\end{array}$} & \multirow[b]{3}{*}{ BAREMO } \\
\hline & & \multicolumn{2}{|c|}{ SI } & \multicolumn{2}{|c|}{ NO } & \multicolumn{2}{|c|}{ Total } & \\
\hline & Ítem & $\mathrm{Fa}$ & $\mathrm{Fr}$ & $\mathrm{Fa}$ & $\mathrm{Fr}$ & $\mathrm{n}$ & $\%$ & \\
\hline 21 & $\begin{array}{l}\text { ¿Evalúan la eficiencia de la política de } \\
\text { mantenimiento que se ha planificado? }\end{array}$ & 8 & 53,33 & 7 & 46,67 & 15 & 100 & Moderada presencia \\
\hline 22 & $\begin{array}{l}\text { ¿̇ldentifican las áreas en que se tienen } \\
\text { deficiencias circunstanciales? }\end{array}$ & 7 & 46,67 & 8 & 53,33 & 15 & 100 & Moderada presencia \\
\hline 23 & $\begin{array}{l}\text { ¿La dirección establece rutas de acción } \\
\text { para erradicar debilidades detectadas? }\end{array}$ & 6 & 40,00 & 9 & 60,00 & 15 & 100 & Moderada presencia \\
\hline 24 & $\begin{array}{l}\text { ¿Evalúan el desempeño del personal } \\
\text { que constituye la unidad de } \\
\text { mantenimiento? }\end{array}$ & 12 & 80,00 & 3 & 20,00 & 15 & 100 & $\begin{array}{c}\text { Alta } \\
\text { presencia }\end{array}$ \\
\hline 25 & $\begin{array}{l}\text { ¿Realizan evaluaciones cíclicas donde se } \\
\text { identifiquen desviaciones para plantear } \\
\text { soluciones a las mismas? }\end{array}$ & 6 & 40,00 & 9 & 60,00 & 15 & 100 & Moderada presencia \\
\hline & Totales & 8 & 52,00 & 7 & 48,00 & 15 & 100 & Moderada presencia \\
\hline
\end{tabular}

Fuente: Elaboración propia 
Estos resultados reflejan que, en las unidades de bombeo analizadas, las siguientes actividades ostentan moderada presencia: evalúan la eficiencia de la política de mantenimiento que se ha planificado $(53,33 \%)$; identifican las áreas en que se tienen deficiencias circunstanciales $(46,67 \%)$; la dirección establece rutas de acción para erradicar debilidades detectadas (40\%); y realizan evaluaciones cíclicas donde se identifiquen desviaciones para plantear soluciones a las mismas (40\%). No obstante, se categoriza con alta presencia solo una actividad de la evaluación referida a si: evalúan el desempeño del personal que constituye la unidad de mantenimiento (80\%).

Los resultados evidenciados validan medianamente los postulados teóricos de Acosta (2007), cuando indica que se debe evaluar la eficiencia de la política de mantenimiento que se ha planificado para el entorno productivo de una organización, e identificar las áreas en que tiene deficiencias estructurales y circunstanciales, lo cual es un aporte sustancioso puesto que la dirección puede establecer una ruta de acción para erradicar las debilidades detectadas.

A juicio de la investigadora, las unidades de bombeo bajo estudio no están brindando la importancia de la evaluación en la gestión de mantenimiento que llevan a cabo, obviando que la aplicación periódica de instrumentos de evaluación les permite detectar cómo responde la organización ante las variaciones a las que está sujeta y de qué forma debe adecuarse. Restando valor al hecho de que las evaluaciones forman parte de revisiones cíclicas y sistemáticas, donde se identifican desviaciones y se plantean soluciones a las mismas.

Para la dimensión elementos de la gestión de mantenimiento, cuyos resultados se concentran en la Tabla 7, se observa cómo el $73,33 \%$, en promedio, de los encuestados consideraron la opción afirmativa, indicando alta presencia de estos elementos en la gestión de mantenimiento que desarrollan las unidades de bombeo mecánico convencionales en Petróleos de Venezuela división Costa Oriental del Lago.

Tabla 7. Dimensión: Elementos de la gestión de mantenimiento

\begin{tabular}{lccccccc}
\hline \multicolumn{7}{c}{ ESCALA DICOTÓMICA } \\
INDICADOR & $\mathrm{Fa}$ & $\%$ & $\mathrm{Fa}$ & $\%$ & $\mathrm{n}$ & $\%$ & BAREMO FRECUENCIA \\
\hline Planificación & 13 & 89,33 & 2 & 10,67 & 15 & 100 & Alta presencia \\
Programación & 10 & 65,33 & 5 & 34,67 & 15 & 100 & Moderada presencia \\
Ejecución & 12 & 80,00 & 3 & 20,00 & 15 & 100 & Alta presencia \\
Control & 12 & 80,00 & 3 & 20,00 & 15 & 100 & Alta presencia \\
Evaluación & 8 & 52,00 & 7 & 48,00 & 15 & 100 & Moderada presencia \\
\multicolumn{1}{c}{ DIMENSIÓN } & 11 & 73,33 & 4 & 26,67 & 15 & 100 & Alta presencia \\
\hline
\end{tabular}

Fuente: Elaboración propia 
Al detalle se aprecia que la planificación, ejecución y control son considerados con alta presencia al momento de gestionar el mantenimiento. No obstante, la programación y evaluación muestran oportunidades de mejora dado que alcanzan la categoría de moderada presencia, según el baremo utilizado.

Estos resultados, de alta presencia de la dimensión, logran validar la teoría expuesta por Martínez (2007), para quien los elementos de la gestión de mantenimiento, como toda gestión organizacional, están relacionados a un sistema de dirección que se base en la planificación, organización, programación, control y ejecución. Bajo esta óptica la gestión de mantenimiento es un ciclo que se da en forma secuencial.

También alcanzan alta congruencia con Márquez (2010), para quien los elementos de la gestión de mantenimiento expresan el trabajo de planificar, organizar, programar, ejecutar, controlar y evaluar, a realizarse para maximizar tanto la disponibilidad como efectividad de la infraestructura requerida por el sistema de producción.

Afirmando, además, la posición de Prando (2007), cuando explica que la gestión de mantenimiento es un proceso integral basado en normas y procedimientos, en el cual se distribuyen las acciones de la gerencia de línea. Enfatiza que el mantenimiento como proceso se realiza a nivel operativo, pero como gestión, se sustenta en la alta y media gerencia a través de los procesos de planificación, control y supervisión.

Para cerrar, la investigadora observa que, en las unidades de bombeo mecánico convencionales en Petróleos de Venezuela división Costa Oriental del Lago se asigna alta presencia a los elementos claves de la gestión de mantenimiento, desde la óptica de los autores que fundamentan la teoría de esta investigación.
Sin embargo, se muestran oportunidades de mejora en todos los elementos medidos en esta dimensión, dado que se evidenciaron en cada uno de ellos actividades que se desarrollan con moderada presencia, las cuales serán objeto de análisis en el objetivo referido a establecer acciones estratégicas que orienten esta gestión.

La idea central es que, quien gestiona el mantenimiento cuente con las herramientas brindadas en este trabajo de investigación para dar a el nivel de presencia máximo a los elementos de la gestión de mantenimiento, viéndolos como el conjunto de acciones pertinentes para su cumplimiento, que contribuyan a la efectividad de los procesos productivos, habiendo definido estas acciones como: planificación, organización, programación, ejecución, control y evaluación.

\section{CONCLUSIONES}

Se identificaron los elementos de la gestión de mantenimiento presentes en las unidades de bombeo mecánico convencionales en Petróleos de Venezuela división Costa Oriental del Lago, se concluye que éstos poseen alta presencia en la gestión de mantenimiento analizada, señalando como elementos de la gestión la planificación, programación, ejecución, control y evaluación, es decir, el conjunto de acciones pertinentes para su cumplimiento.

Al respecto se tienen las siguientes conclusiones: la planificación, ejecución y control son considerados con alta presencia al momento de gestionar el mantenimiento; no obstante, la programación y evaluación muestran oportunidades de mejora dado que alcanzan la categoría de moderada presencia, esto indica que en estas unidades se planifica, ejecuta y controla el mantenimiento de manera adecuada; pero se deben revisar las acciones que se realizan para programar y evaluar el mismo. 
REFERECIAS

Acosta, H. (2007). El control y la evaluación de la gestión del mantenimiento. [Nota técnica en línea]. Ciudad de la Habana: Coordinación Editorial CEIM-CUJAE. Disponible en: http://www.cujae.edu.cu

Adam, E. y Ebert, R. (2006). Administración de la producción y operaciones, conceptos, modelos y funcionamiento. Cuarta Edición. Boston, Universidad de Arizona. Estados Unidos de América

Dounce, E. (2008). La Productividad en el mantenimiento industrial. Editorial C.E.C.S.A. México

Duffuaa, S.; Raouf, A; Campbell, J. (2010). Sistemas de mantenimiento. Planificación y control. Editorial Limusa. México

Fundación Educación Industrias (Fundei) (2009). La gestión del mantenimiento. Material de curso. Venezuela

García, S. (2009). Organización y gestión integral de mantenimiento. Tercera Edición. Ediciones Díaz de Santos S.A. Madrid. España

Márquez, M. (2010). Manual de la ingeniería de calidad. Gestión de mantenimiento. Editorial Panapo. Caracas. Venezuela

Martínez, L. (2007). Organización y planificación de sistemas de mantenimiento. Centro de altos estudios gerenciales. Instituto Superior de Investigación y Desarrollo. Segunda Edición. Caracas. Venezuela

Moreno, P. (2012). Auditoria del mantenimiento e indicadores de gestión. Segunda edición. FC editorial. Madrid, España
Neto, E. (2008). Mantenimiento industrial. Seminario planeación y mantenimiento, Escuela Politécnica Nacional. MacasEcuador

Newbrough, E. (2005). Administración del mantenimiento industrial. Editorial Diana, México.

Norma Venezolana COVENIN 2500-93. Manual para evaluar los sistemas de mantenimiento en la industria. Comité Técnico de Normalización CT-3 Construcción, aprobada por la COVENIN en su reunión № 124 de fecha 93-12-01. Sustituye totalmente a la Norma Venezolana COVENIN 2500. 89. Venezuela

PDVSA (Petróleos de Venezuela, S.A.) (2005). Manual de Mantenimiento. Niveles de Mantenimiento. Caracas. Venezuela

Perozo, A. (2007). Mantenimiento industrial. Cátedra de mantenimiento industrial. Universidad del Zulia. Maracaibo, Venezuela

Prando, R. (2006). Manual de gestión de mantenimiento a la medida. Quinta edición. Editorial Piedra Santa. San Salvador. El Salvador

Rodríguez, P. (2008). Manual de mantenimiento a la medida. FC Editorial. España

Souris, J. (2005). El mantenimiento, fuente de beneficios. Octava edición. Ediciones Díaz de Santos. Madrid. España 